\title{
Characterization of Reflective TN-LCD, Tuned in Phase-Only Modulation and to Six Wavelengths
}

\author{
Jorge Enrique Rueda, Ana Ludia Romero ${ }^{1}$, Luis Alfonso Guerra ${ }^{2}$ \\ Grupo Óptica Moderna, Universidad de Pamplona, Pamplona-Colombia, A.A.1046. \\ ${ }^{1,2}$ Master of Physics Students, Departamento de Física, UniPamplona, Colombia.
}

Received November 22, 2010; accepted November 29, 2010; published December 31, 2010

\begin{abstract}
We present the results of the characterization of a liquid crystal spatial modulator LCR2500. The phase modulation ratio versus applied voltage and six wavelengths was determined. The basic experimental arrangement is a Mach-Zehnder interferometer, where one mirror is a LCR2500. We found the modulation exceeding $2 \pi$ radians. The method eliminates errors in the phase due to a mechanical noise.
\end{abstract}

The spatial light modulators are liquid crystal arrays of opto-electronic cells digitally controlled from a computer. The birefringence caused for an electro-optical effect in the device allows its use in spatial manipulation of the properties of polarization, amplitude and phase of a wavefront. A particular interest in the use of these devices is the spatial modulation of phase and/or amplitude of the wavefront. From the modulation of the wavefront different possible applications are, among others, encoding holographic filters and diffractive optical. In this direction, it is necessary to specify the characterization of these modulators. Several authors have presented methods to characterize liquid crystal display of transmission and reflection [1]-[17].

One way to tune a liquid crystal modulator in a single phase modulation is to ensure that the incident wave in the modulator is linearly polarized and that this same state of polarization is oriented parallelly to the principal axis of the modulator. Under this condition, the extraordinary refractive index depends on the applied voltage, and the plane of polarization of light reflected or transmitted from one liquid crystal modulator only suffers a rotation, so that the linear polarization state is preserved. Such dependence of the extraordinary refractive index implies that the light reflected or transmitted, also changes the speed of propagation, and therefore, phase-only modulation can be possible. The difficulty in achieving harmony in one phase modulation depends on how accurately we establish the condition of parallelism between the state of linear polarization of the incident wave and the director axis of the modulator.

This paper is a report of the characterization results of a LCR2500, tuned phase-only modulation for six wavelengths. To determine the response of the phase-only modulation, we use a Mach-Zehnder interferometer, in which one mirror is a LCR2500, where the interference pattern generated has two fully defined zones, which correspond to two phase planes generated in the modulator. Then the module is divided into two zones, both of constant gray level, which we call phase planes. One zone is the reference phase plane (Plane Zone II = gray level zero) and in the other zone (Zone I) changes the gray level from zero to 255 , for a total of 256 planes of phase.

This interferometer has been suggested by other authors for the same purpose to characterize liquid crystal modulators. However, we propose an alternative mode of operation and also in the treatment of the information to determine the phase. So, to tune the modulator in single phase, we apply to Zone I gray level 255 and to Zone II gray level zero, having done that and with the help of a half-wave retarder, we proceed to rotate the polarization plane incident light modulator to obtain the maximum relative lateral shift between the two areas of interference. The next step is to adjust for Zone I, one by one, the 256 planes of the respective phase and determine the relative lateral shift between the two areas of interference.

The experimental setup is shown in Fig. 1. In this Mach-Zehnder interferometer one of the mirrors is the LCR2500. Holoeye LCR2500 system is a screen reflective TN-LCD-Twisted Nematic Liquid Crystal Display-45 ${ }^{\circ}$, monochromatic, XGA resolution $1024(\mathrm{H})$ x768 (V) pixels, the modulation function is electrically controlled via DVI port, cell size is $19 \mu \mathrm{m}$. The light source arrangement is Argon laser multi-line $(457 \mathrm{~nm}$ : 15mW, 476nm: 19mW, 488nm: 40mW, 496nm: 19mW, 501nm: 40mW, 514nm: 19mW).

The linear polarizer $\mathrm{P}$ and the two half-wave retarders were arranged on rotating mounts precision 1/200 degrees. The Motic352 CCD camera is $800 \times 600$ pixels resolution.

The retarder $\mathrm{R} 1$ function is to manipulate the orientation of the plane of polarization about the director of the modulator; in our case, the orientation of linear polarization with respect to the liquid crystal director was $-3^{\circ}$. The retarder $\mathrm{R} 2$ is used to control the maximum visibility of the interference pattern. 


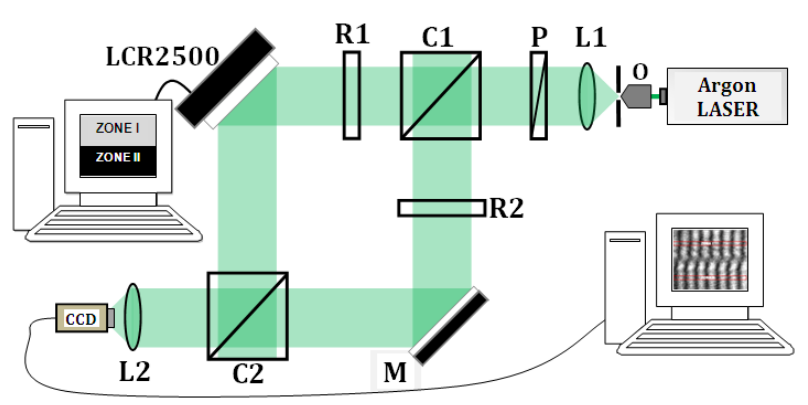

Fig. 1. Experimental setup. L1: $+30 \mathrm{~cm}$ focal length lens, L2: lens focal length $+20 \mathrm{~cm}, \mathrm{C} 1,2$ : Cubic Beam splitters, M: mirror, O: microscope objective; R1,2: half-wave retarders, P: linear polarizer.

Based on the models presented in references [15]-[16], the model of experimental arrangement utilized is:

$$
\left|E_{\text {out }}\right\rangle=M_{L C R} R 1|E\rangle+R 2|E\rangle
$$

The first term represents the beam passing through the modulator. Being $\mathrm{M}_{\mathrm{LCR}}$ liquid crystal modulators, $\mathrm{R} 1$ and $\mathrm{R} 2$ are half-wave retarders and $\mathrm{E}$ is a linearly polarized radiation field. The Jones's matrix of the modulator is:

$$
\begin{gathered}
M_{\text {LCR }}=\exp \left(\frac{i \Gamma)}{2}\right) \cdot\left(\begin{array}{cc}
\Phi \frac{\sin \chi}{\chi} & \cos \chi-\mathrm{i} \frac{\Gamma}{2} \frac{\sin \chi}{\chi} \\
-\cos \chi+\mathrm{i} \frac{\Gamma}{2} \frac{\sin \chi}{\chi} & \Phi \frac{\sin \chi}{\chi}
\end{array}\right) \\
\Gamma(\theta)=\frac{2 \pi}{\lambda}\left[n_{e}(\theta)-n_{o}\right] d
\end{gathered}
$$

Where $\Gamma$ is the phase introduced by the modulator, $\chi$ (twist angle) is the angle of rotation of the liquid crystal director (axis of rotation is the direction of propagation), and $\theta$ is the tilt angle of the liquid crystal director, caused by the applied voltage (or gray level). $n_{\mathrm{e}}$ and $n_{\mathrm{o}}$ are the extraordinary and ordinary indices of the crystal, $d$ is the thickness of glass, $\lambda$ is the wavelength of light, and $\Phi$ is the total angle that rotates the optical axis.

As for the mode of operation, it is exactly as it is described in the introduction; where the basic idea is to divide the line into two modulator voltage zones, so that half of the modulator is active with zero applied voltage or gray level zero (Zone II of reference) and the other half will change the voltage or gray level from zero to 255 (see Fig. 1). In Fig. 2, we show an example of such an effect, after applying to Zone I a gray level 137, and generated a phase shift of 3.94 radians between the two areas of the interference pattern. Thus, after digital image processing of each interference pattern, we determined the phase introduced by each gray level applied to the Zone I and for each of the lengths of multi-line laser used. The phase shift between the Zone I and II, was determined from the data enclosed by the red box (see Fig. 2), so, we made two cuts of $21 \times 371$ pixels, one for each Zone. Figure 3 shows the cuts made to each Zone of the digitally processed image (Fig. $2 b$ ). To improve the outcome of this measure, given the high level of noise in the images recorded by the $\mathrm{CCD}$, we implemented a method of digital image restoration, based on a Fourier transform [18]. In Fig. 2b and Fig. 3 they show the result of digital processing applied to the image in Fig. 2a.

In Fig. 4 it shows the behaviour of the intensity determined for a lighting of $514 \mathrm{~nm}$, the same behaviour was observed for the other five lengths of the laser used.

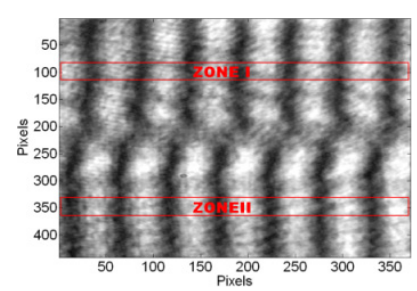

(a)

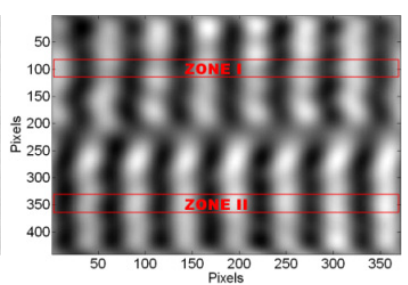

(b)
Fig. 2. Interference pattern image, after applying the gray level 137 in Zone I, without filtering. (b). Image (a) after processed digitally.

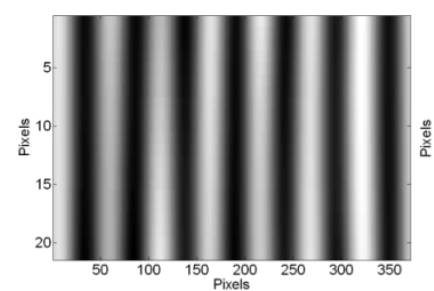

(a)

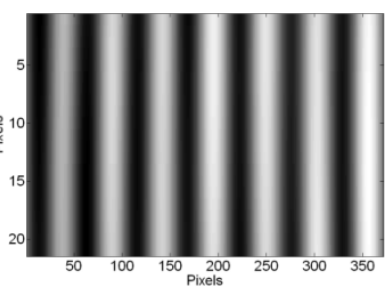

(b)
Fig. 3. Cuts of the image of figure 2b. (a). Cut of the Zone I; (b). Cut of the Zone II.

The results shown in Table 1 are the maximum modulation for each wavelength. It also lists the gray level values for each wavelength, producing phase shifts $\pi$ and $2 \pi$. We found that for the $488 \mathrm{~nm}$ wavelength it is not possible to obtain a $2 \pi$ phase shift.

Table 1 . Grey level modulation $\pi$ and $2 \pi$, and maximum modulation.

\begin{tabular}{cccc}
\hline$\lambda(\mathrm{nm})$ & $\begin{array}{c}(\text { Phase } \pi) \\
\text { GL }\end{array}$ & $\begin{array}{c}\text { (Phase } 2 \pi) \\
\text { GL }\end{array}$ & $\begin{array}{c}\text { (GL 255) } \\
\text { Phase (rad) }\end{array}$ \\
\hline 514 & 184 & 254 & 7.127 \\
501 & 166 & 254 & 6.618 \\
493 & 164 & 243 & 6.900 \\
488 & 150 & Off Set & 5.884 \\
476 & 164 & 255 & 6.610 \\
457 & 146 & 234 & 7.127 \\
\hline
\end{tabular}




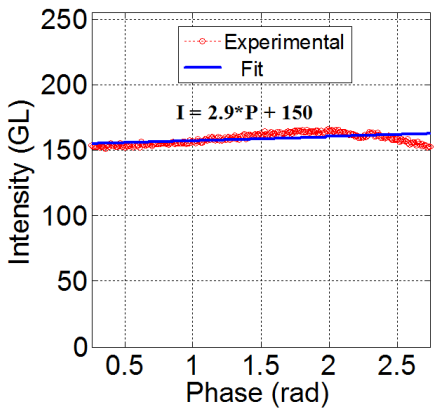

Fig. 4. Variation in the intensity of the interference pattern for $\lambda=514 \mathrm{~nm}$ STD: 0.024 GL; I: Intensity; P: phase.
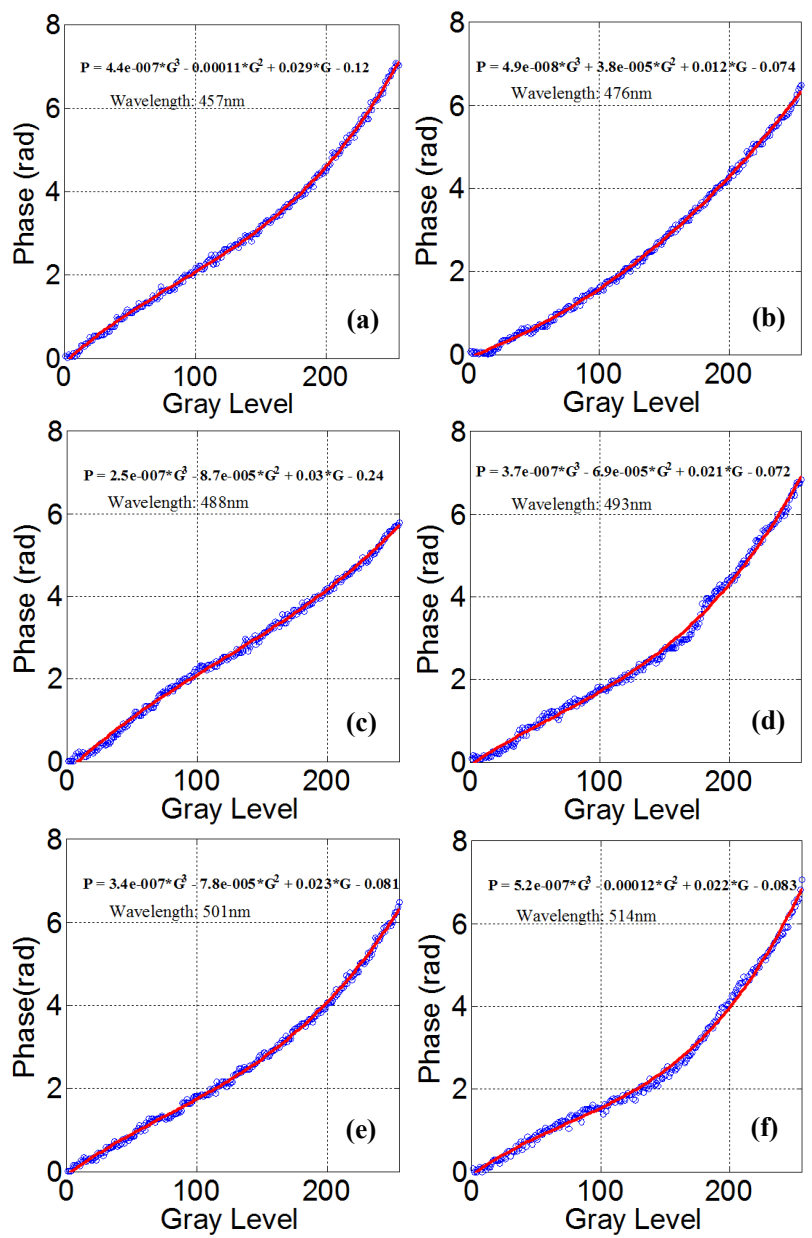

Fig. 5. Phase modulation vs. Gray levels. P: Phase; G: Gray Level.
The results of phase-only modulation vs. gray levels, and six wavelengths are shown in Fig. 5. In the adjustment functions $P(G)$, $P$ represents the phase and $G$ gray level applied to the Zone I.

By implementing a Mach-Zehnder interferometer, operating under a condition of high mechanical vibration of the optical bench, we show that the modulation method in areas of the same interference pattern has a high confidence factor in determining the characteristics of phase modulation functions of LCR2500. We determine the phase modulation functions $\mathrm{P}(\mathrm{G})$ for six wavelengths. On the other hand, we find that the method guarantees a minimum phase-amplitude coupling; the behaviour can be inferred from Fig. 4, where the variation of a coupling is about $1 / 100$. Another advantage of the method employed is the direct visualization of a phase shift while changing from the gray level. Similarly, we found that the method facilitates tune modulation points of maximum phase, by direct visualization of the interference pattern. We use the phase function $\mathrm{P}(\mathrm{G})$ to determine the values listed in Table 1 .

\section{References}

[1] E. Badosa, et al., Meas. Sci. Technol. 8, 764 (1997).

[2] I. Moreno, et al., Opt. Eng. 37, 3048 (1998).

[3] T. Kelly, et al., Opt. Comm. 156, 252 (1998).

[4] A. Lizana, et al., Opt. Exp. 16(21), 16721. (2008).

[5] J. Coy, M. Zaldarriaga, D. Grosz, O. Martínez, Opt. Eng. 35, 15 (1996).

[6] A. Márquez, et al., Opt. Eng. 39, 3301 (2000).

[7] J. Davis, D. Allison, K. D’Nelly, I. Moreno, Opt. Eng. 38, 705 (1999).

[8] J. Pezzaniti, R. Chipman, Opt. Lett. 18, 1567 (1993).

[9] J. Davis, I. Moreno, P. Tsai, Appl. Opt. 37(5), 937 (1998).

[10] A. Márquez, et al., Opt. Eng. 40, 2558 ( 2001).

[11] J. Nicolás, J. Campos, J. Yzuel, Opt. Soc. Am. A 19, 1013 (2002).

[12] A. Márquez, C. Cazorla, M. Yzuel, J. Campos, J. Mod. Opt. 52, 633 (2005).

[13] L. Bougrenet, L. Dupont, Appl. Opt. 36, 1730 (1997).

[14] D. Berreman, J. Opt. Soc. Am 63, 1374 (1973).

[15] K. Lu, B. Saleh, Opt. Eng. 29, 240 (1990).

[16] A. Yariv, P. Yeh, Optical waves in crystals (New York, John Wiley \& Sons 1984).

[17] I. Khoo, Liquid crystals (New York, John Wiley \& Sons 2007).

[18] Takeda, et al., J. Opt. Soc. Am. 72, 156 (1982). 